Military Technical College Kobry El-Kobbah, Cairo, Egypt

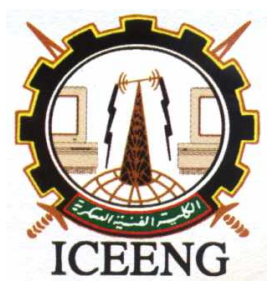

\author{
$6^{\text {th }}$ International Conference \\ on Electrical Engineering \\ ICEENG 2008
}

\title{
Multi-user detection based on neural network for multi-carrier code division multiple access systems
}

By

Necmi Taşpınar*

Metin Çiçek**

Yalçın Iş1k***

\section{Abstract:}

In this paper, we present fundemental linear multiuser detection (MUD) techniques and compare them with the technique based on neural network (NN) in multicarrier code division multiple access (MC-CDMA) systems. In a MC-CDMA system, increasing with the number of users, receiver's bit error rate (BER) performance goes up. Also, the system' s performance is effected by the power level differences among the users. Simulation results demostrate the higher performance of NN receiver compared to conventional receiver (matched filter) for MC-CDMA. And also, performance results show that the NN structure, gives nearer results comparing to decorrelator and MMSE receivers. Simulations implemented in MATLAB program and performances are examined for synchronous communication and AWGN channels. The LevenbergMarquardt algorithm is used as the learning algorithm for NN.

\section{Keywords:}

Multiuser detection (MUD), MC-CDMA, neural network

* Department of Electrical and Electronic Engineering, Erciyes University, Kayseri, Turkey

** Graduate School of Natural and Applied Sciences, Erciyes University, Kayseri, Turkey

*** Silifke-Taşucu Vocational High School, Selçuk University, İçel, Turkey 


\section{Introduction:}

The new multimedia services defined in the $3 \mathrm{G}$ and $4 \mathrm{G}$ will require high data rates over broadband radio channels. In order to meet the requirements of those applications, multiple Access (MC) techniques should offer high spectral efficiency and flexibility. Spread spectrum has been adopted for the UMTS and IMT2000, by using the Code Division Multiple Access (CDMA) capabilities [1]. On the other hand, multi-carriers, well known under the acronym Orthogonal Frequency Division Multiplexing (OFDM), has been receiving interest for the same application. Two techniques can be combined, under MC-CDMA modulation. MC-CDMA benefits from the advantages of both schemes: high spectral efficiency, multiple access, and interference rejection. In a CDMA system, several users simultaneously transmit information over a common channel using pre-assigned codes. By selecting mutually orthogonal spreading codes for all users, they each achieve interference-free single-user performance. It is however not possible in a mobile environment to maintain orthogonality of the codes at the receiver. The conventional matched filter detector consists of a bank of filters matched to the spreading codes. This detector suffers from two problems: First, multiple access interference (MAI) produced by the other co-channel users is a significant limitation to the capacity of this detector [2]. The second problem is the near-far effect which occurs when the relative received power of interfering signals becomes larger. A lot of multiuser detection (MUD) techniques has been proposed in literature[3-4]. A potential solution of these problems is the optimum multiuser detector (MUD) that consists of a bank of matched filters followed by a Viterbi maximum likelihood (ML) detector [5-6]. However the computational complexity of this detector increases exponentially with the number of users, and the method is extremely complex to implement for a realistic number of users. Therefore, there has been considerable research into suboptimal detectors. Two types of linear detectors have also been suggested. These are decorrelating detector [7] and the minimum mean squared error (MMSE) detector [8-9]. Complexity of these receivers is linear in the number of users. In addition to linearMUD techniques, there are many of nonlinear MUD techniques. One of these techniques is detection based on neural network (NN) structure. Artificial neural network (ANN) was firstly used for multiuser detection in 1992 by Aazhang, Paris and Orsak[10]. Then, that study became a reference for many of the studies[11-12-13].

In this paper, we compare the performances of the linear multiuser detection techniques with the non-linear multi user detection technique based on neural network $(\mathrm{NN})$ in MC-CDMA systems by computer simulations. Simulations were performed in three different ways : The BER of the desired user versus the signal-to-noise ratio (SNR), the BER of the desired user versus the number of user in the system and the BER of the 
desired user versus the near-far rate (NFR).

\section{MC-CDMA system model:}

Figure (1) shows K-user synchronous transmitter system model. Transmitted signal of CDMA user's can be expressed as:

$$
x(t)=\sum_{n=1}^{L} A_{k} b_{k} s_{k, n} e^{j w_{n} t}
$$

where $b_{k}$ is the input data for $\mathrm{k}$ th user, $\mathrm{A}_{\mathrm{k}}$ is the amplitude of $k$ th user. The signal received at the end of the receiver is denoted as:

$r(t)=\sum_{k=1}^{K} \sum_{n=1}^{L} A_{k} b_{k} s_{k, n} e^{j w_{n} t}+n(t)$

where $n(t)$ denotes an additive white Gaussian noise (AWGN) and $s_{k}$ is the signature waveform of kth user. $s_{k}$ is defined for $\mathrm{L}$ signature sequence length and BPSK modulation as:

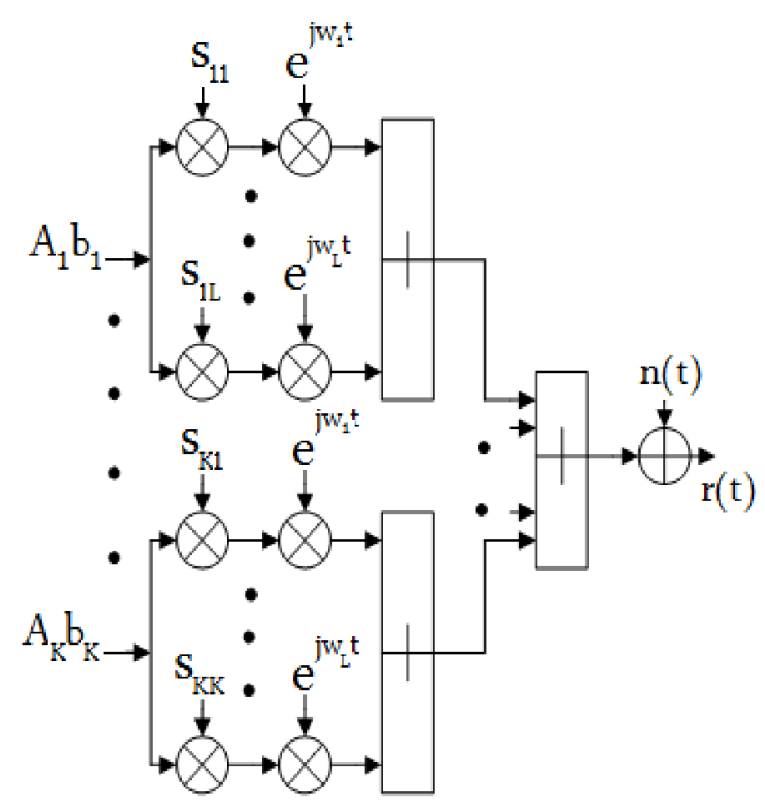

Figure (1): K-user synchronous transmitter system model. 
$\mathrm{s}_{\mathrm{k}}(\mathrm{t})=\sum_{\mathrm{n}=0}^{\mathrm{L}-1} \mathrm{a}_{\mathrm{k}} \mathrm{p}_{\mathrm{T}}\left(\mathrm{t}-\mathrm{nT} \mathrm{T}_{\mathrm{c}}\right)$

where $a_{k}$ is the normalized spreading sequence, $T_{c}$ is the chip interval, $p_{T}$ is the rectangular waveform of the duration $\mathrm{T}_{\mathrm{c}}$, and $\mathrm{T}=\mathrm{LT}_{\mathrm{c}}$.

\subsection{Receiver:}

Figure (2) shows the receiver diagram of a MC-CDMA system. As shown in Fig.2, the signal taken from channel is coppied to L parallel channels and FFT operation is performed over that signal. After that, FFT outputs are applied to the detection scheme.

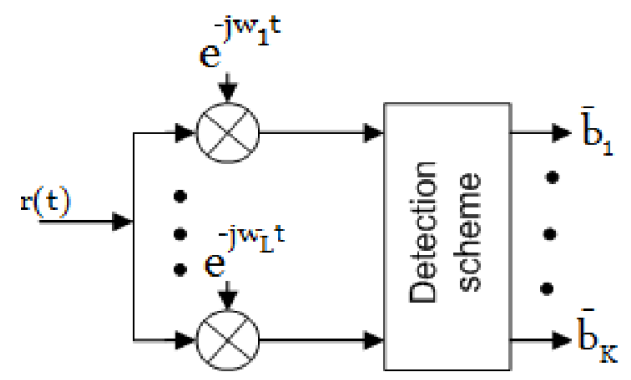

Figure (2): $M C$-CDMA receiver.

\subsubsection{Matched Filter:}

One of the most common approches to multiuser detection (MUD) in MC-CDMA is maximum ratio combining (MRC) technique. This technique is performed with using matched filter. Figure (3) shows the receiver diagram of MUD using matched filter.

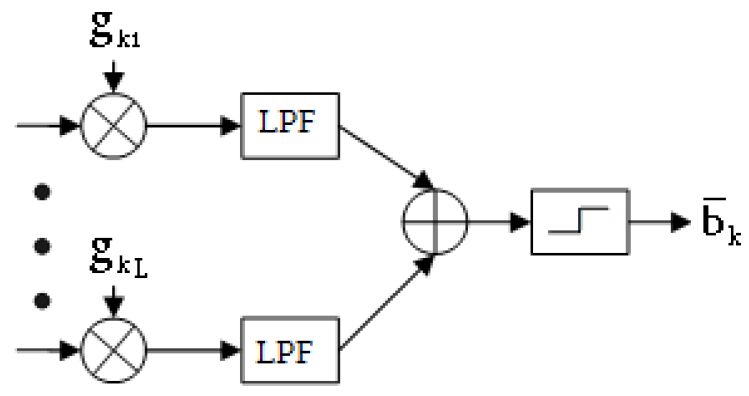

Figure (3): Matched filter. 
In the receiver, FFT outputs are applied to matched filter's inputs. $g_{k[i]}=\left[g_{k 1} g_{k 2} \mathrm{~K} g_{k L}\right]$ is the subcarrier signal's weighting factor for user $k$. Durability of this structure to near-far problem is poor and BER performance becomes high when the number of users increase.

\subsubsection{Decorrelating Detector:}

Figure (4) shows the multiuser detection (MUD) with decorrelating detector. The decorrelating detector applies the inverse of the correlation matrix of the spreading codes to the matched filter outputs, Y.

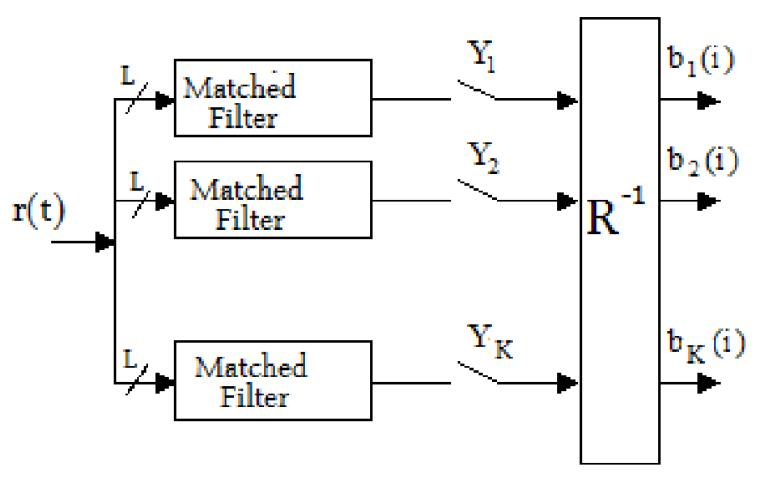

Figure (4): Decorrelating detector.

Detector's output is

$\overline{\mathrm{b}}_{\mathrm{decor}}=\mathrm{R}^{-1} \mathrm{Y}=\mathrm{Ab}+\mathrm{R}^{-1} \mathrm{n}$

Different from the matched filter, as correlation matrix of the spreading codes regarded, even increasing of the number of the users or the near-far ratio (NFR); it gives better performance.

\subsubsection{MMSE detector:}

The minimum mean-squared error multiuser detector is a linear detector which takes into account the background noise and utilizes knowledge of the received signal powers.

In this detector, $\left(R+N_{0} A^{-2}\right)^{-1}$ function is applied to the matched filter outputs. We can define, 
$\mathrm{N}_{0} \mathrm{~A}^{-2}=\operatorname{diagonal}\left\{\frac{\mathrm{N}_{0}}{\mathrm{~A}_{1}{ }^{2}}, \frac{\mathrm{N}_{0}}{\mathrm{~A}_{2}{ }^{2}}, \mathrm{~K}, \frac{\mathrm{N}_{0}}{\mathrm{~A}_{\mathrm{K}}{ }^{2}}\right\}$

where $N_{0}$ is noise power and $A$ is the signal amplitude matrix. In this structure, because of taking into account the noise factor, it gives good performance.

\subsubsection{Artificial Neural Networks (ANNs):}

Neural network (NN) detection scheme is shown in Figure (5) for 2-users. Neural Networks are constructed with neurons that connected to each other. The NN consist of input, hidden and output layers and they have forward connections between neurons. Each connection has a weight factor and these weights are adjusted in a training process. Weights are changed with various learning algorithm to get a proper output.

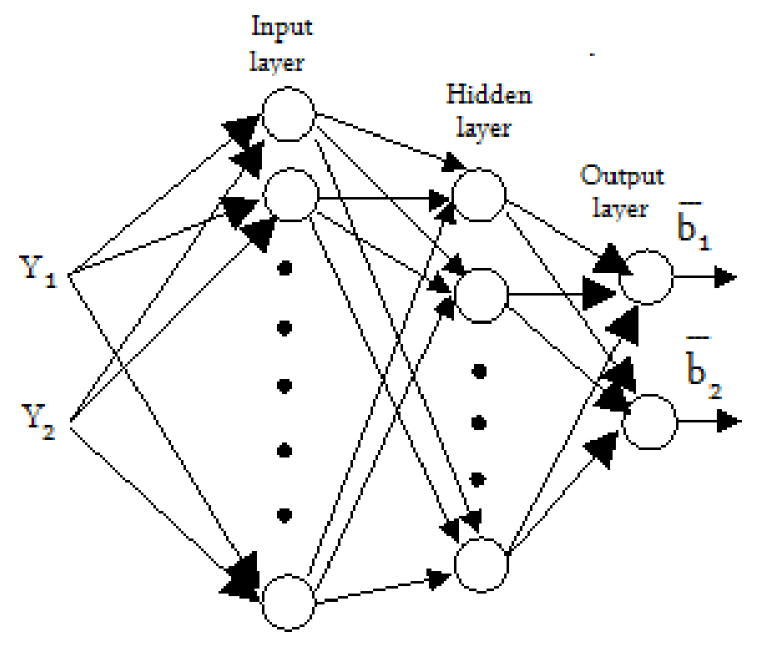

Figure (5): The neural network (NN) structure.

\section{Simulation Results:}

Simulations were performed in three different ways : The bit error rate (BER) of the desired user versus the signal-to-noise ratio (SNR), the BER of the desired user versus the near-far rate (NFR) and the BER of the desired user versus the number of user in the system. In all simulations, a synchronous additive white Gaussian channel (AWGN) 
channel is considered and 31 bits length spreading codes with normalized crosscorrelation 0.226 between all codes are used. The NN is trained with data of 1000 bits. The number of input nodes, hidden layer nodes and output nodes of the structure of NN is equal to the number of active users. It is a feed forward network and it is trained by Levenberg-Marquardt algorithm. In the 5 users synchronous AWGN channel, the BER values of the first user versus SNR for various multiuser receivers are shown in Fig.6. In Figure (6), the performance superiority of the neural network receiver over matched filter can be seen clearly. As it is seen, the MMSE detector, decorrelating detector and NN receiver provide similar performances. In Figure (7), there are 10 users in the system and it is seen that the performances of the all receiver structures is got worse. Increasing with the number of users, receiver's bit error rate (BER) goes up for all receiver structures. Figure (8) shows the bit error rate of first user for the number of active users in the system. In that situation, signal-to-noise ratio is assumed $5 \mathrm{~dB}$ and the amplitudes of the all users are assumed as equal. It is seen from Fig.8 that when the number of receivers increases, bit error rate (BER) also increases.

The NFR of user $\mathrm{k}$ is defined such as:

$N F R_{k}=\frac{\text { user } k \text { power }}{\text { user } 1 \text { power }}=\frac{A_{k}^{2}}{A_{1}^{2}}$

where $A_{k}$ is the amplitude of the user k's signal. Figure (9) shows the BER performance of the first user versus NFR of the second user, while the other user's NFRs are equal to1. As it is seen, NN structure has good performance to near-far problem. It significantly outperforms the matched filter and gives similar results to other linear MUD techniques.

\section{Conclusion:}

In this study, we present conventional and linear MUD techniques and compare them to proposed neural network $(\mathrm{NN})$ structure. We have got good performance with the NN receiver, which is close to the MMSE and decorrelating detector. Compared with the performance of the conventional receiver (matched filter), the proposed $\mathrm{NN}$ receiver provides better performance.

\section{Acknowledgement}

This study was supported by a grant of the Erciyes University, Scientific Research Projects Unit (No. FBT-06-63), Turkey 


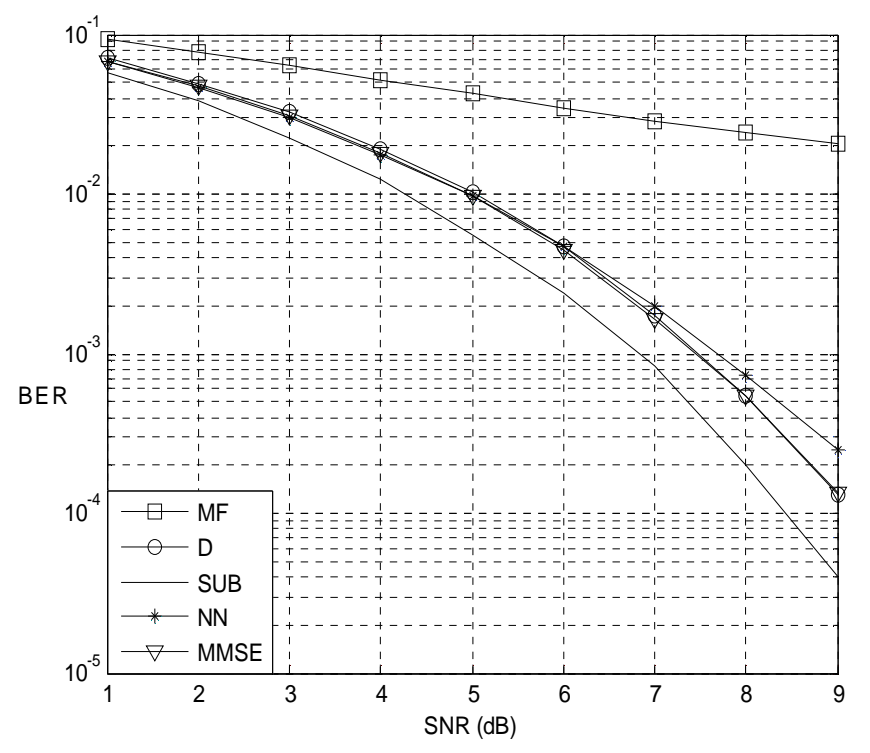

Figure (6): The BER versus the signal-to-noise ratio of the desired user for several detectors in 5-users system.

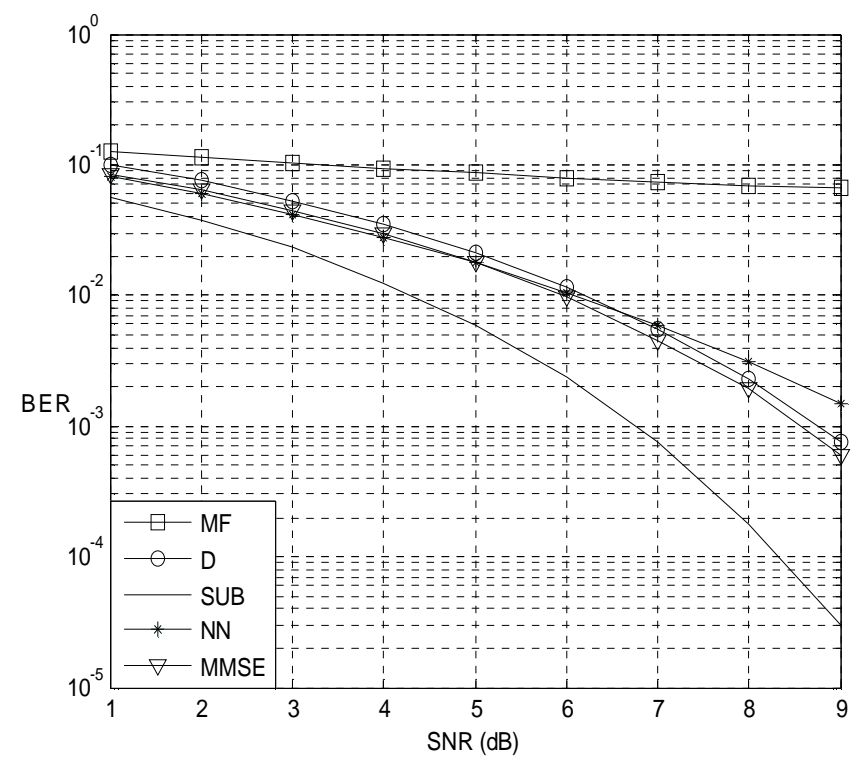

Figure (7): The BER versus the signal-to-noise ratio of the desired user for several detectors in 10-users system. 


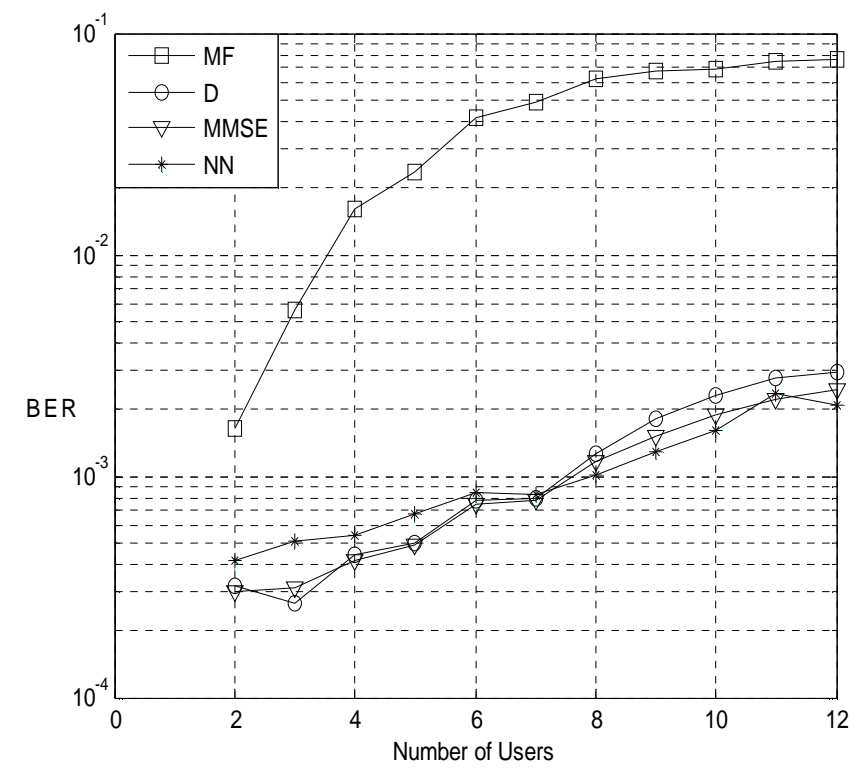

Figure (8): The BER of desired user versus the number of users for several detectors. $(S N R=7 d B)$.

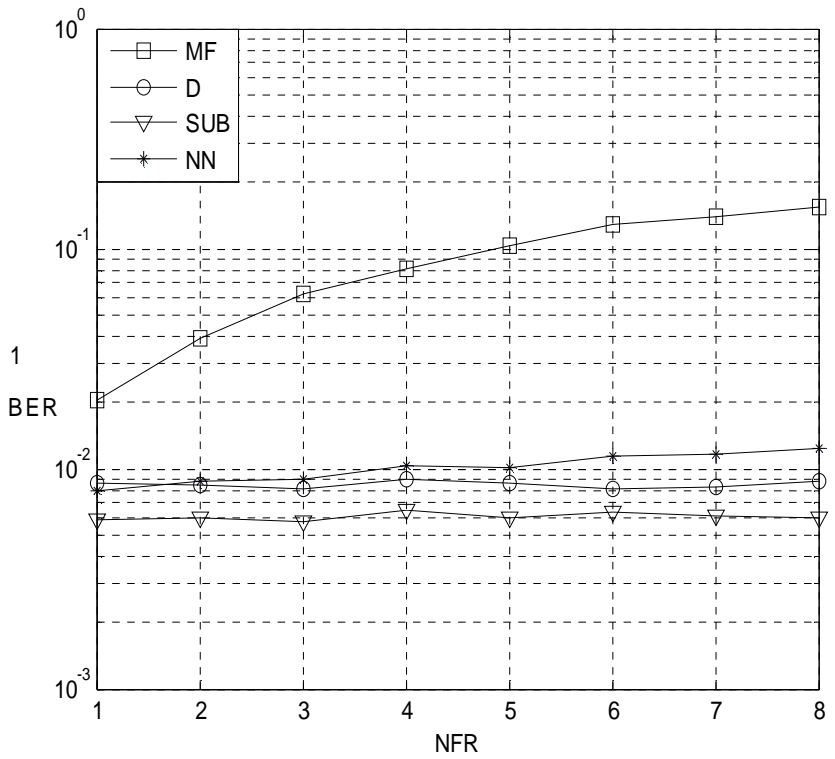

Figure (9): The BER of the desired user versus the near-far rate between the second user $\left(N F R_{2}\right)$ for several detectors. $\left(S N R=5 d B, N F R_{3}=N F R_{4}=N F R_{5}=1\right)$. 


\section{References:}

[1] J.A.C. Bingham, Multicarrier modulation for data transmission: An idea whose time has come, IEEE Communication Magazine, Vol. 36, P. 112-117, February 1998.

[2] S. Hara and R. Prasad, Design and performance of multicarrier CDMA systems in frequency-selective Rayleigh fading channels, IEEE Transaction Vehicular Technology, Vol. 48, P. 1584-1595, September 1999.

[3] S.L. Miller and B.J. Rainbolt, MMSE Detection of Multicarrier CDMA, IEEE Journal on Selected Areas in Communications, Vol. 18, P. 2356-2362, November 2000 .

[4] P. Zong, K. Wang and Y. Bar-Ness, Sampling MMSE Interference Suppression in Asynchronous Multicarrier CDMA Systems, IEEE Journal on Selected Areas in Communications, Vol.19, P. 1605-1613, August 2001.

[5] S. Verdu, Multiuser detection, Cambridge University Press, 1998.

[6] S. Verdu, Minimum probability of error for asynchronous Gaussian multiple-access channels, IEEE Transactions on Information Theory, Vol. 35, P. 123-136, January 1989.

[7] R. Lupas and S. Verdu, Linear multiuser detectors for synchronous code-division multiple-access channels, IEEE Transactions on Information Theory, Vol.35, P.123136, January 1989.

[8] Z. Xie, R.T. Short and C.K. Rushforth, A family of suboptimum detector for coherent multiuser communications, IEEE Journal on Selected Areas Communication, Vol.8, P. 683-690, May 1990.

[9] J-F. Hélard, J-Y. Baudais and J. Citerne, Linear MMSE detection technique for MCCDMA, Electronics Letters, Vol.36, P. 665-666, March 2000.

[10] B. Aazhang, B.P. Paris, G.C. Orsak, Neural networks for Multiuser detection in Code-Division Multiple-Access Communications, IEEE Transactions on 
Communications, Vol. 40, P. 1212-1222, July 1992.

[11] U. Mitra, H.V. Poor, Neural network Techniques for Multi-user Demodulation, Proceedings of the IEEE International Conference on Neural Networks, Vol. 3, P. 1538-1543, 1993.

[12] G. I. Kechriotis, E.S. Manolakos, Hopfield neural network implementation of the optimal CDMA multiuser detector, IEEE Transactions on Neural Networks, Vol. 7, P. 131-41, January 1996.

[13] F. Ibikunle, Y. X. Zhong, Probabilistic neural networks for multi-user detection in code division multiple access communication channels, Proceedings of the IEEE International Joint Conference on Neural Networks and World Computational Intelligence, Vol. 3, P. 2557-2560, 1998.

\section{Nomenclatures:}

$b_{k} \ldots$ Input data for $\mathrm{k}$ th user

$\mathrm{A}_{\mathrm{k}} \cdots \quad$ Amplitude of $k$ th user

$\mathrm{n}(\mathrm{t}) \ldots \quad$ Additive white Gaussian noise (AWGN)

$\mathrm{s}_{\mathrm{k}} \ldots$ Signature waveform of kth user

$a_{k} \cdots \quad$ Normalized spreading sequence

$\mathrm{T}_{\mathrm{c}} \ldots$ The chip interval

$\mathrm{p}_{\mathrm{T}} \ldots$ The rectangular waveform of the duration $\mathrm{T}_{\mathrm{c}}$

$N_{0} \ldots$ Noise power

A... Signal amplitude matrix 